\title{
Bladder Cancer Screening Among Diabetic Patients On Metformin Therapy In A West Africa Subregion
}

\author{
Ufuoma Miller Fakpor ${ }^{1 *}$, Ekpe Ekpe Lawson², Omotoso Ayodele Joshua ${ }^{3}$
}

1. Department Of Medical Laboratory Technology, University Of Cape Coast, Ghana

2. Department Of Chemical Pathology, University Of Calabar, Calabar, Nigeria

3. Department Of Pathology, University Of Calabar, Calabar, Nigeria

\begin{abstract}
Objective: The use of certain medications among diabetic patients put them at risk of developing bladder cancer. This study was done to examine the effects of metformin therapy on the bladder of diabetic patients and to assess the correlation between the effects of metformin and its duration of therapy. Methods: A total of 150 diabetic patients were sampled using a cross sectional study design. $10-15 \mathrm{mls}$ of random urine samples were collected into sterile containers over a period of five weeks. Smears were prepared, fixed and stained using Papanicolaou staining method and examined under the microscope for cytological studies. Results: The probability of developing bladder cancer among diabetic patients on metformin therapy is $0 \%$ among participants of all categories: 99 females and 51 males partook in this study. Peak age group (35.33\%) were between the ages of 51 to 60 years, least age group (4.67\%) were 80 years and above. Traders of food items and used clothes constituted the peak occupational group of $27.52 \%$, with fishermen as least occupation group(0.67\%), age group 61 to 70 had the highest duration of therapy of (15.8\%), the least duration of (4.625\%) was among age group 25 to 30 years, the group of 80 years and above had a duration of $(4.857 \%)$. No inflammatory, degenerative, benign or malignant cells were observed among urine samples from diabetic patients on metformin. Conclusion: This study equips us with knowledge that metformin therapy has no direct influence on the development of cancer of the bladder among diabetic patients as observed through urine cytology. This is the first time such study is done in West Africa region. The clinical implication is that Metformin, the commonest prescribed oral hypoglycemic is safe when considering the carcinogenic effects of anti-diabetic drugs, unlike insulin and some other drugs which have carcinogenic potential.
\end{abstract}

Keywords: metformin; cancer; bladder; diabetes; therapy

\section{Introduction}

Diabetes mellitus (DM) and cancer are very high rate of morbidity and mortality globally. common non-communicable diseases with a Evidences from various studies suggests that

\section{Corresponding author:}

Ufuoma Miller Fakpor

Department Of Medical Laboratory Technology, University Of Cape Coast, Ghana

Email: lawsonekpe2002@yahoo.com

Receive date: 2016-09-7| Accept date: 2016-10-15| Publish date: 2016-11-12

DOI: 10.7575/aiac.abcmed.16.04.04.01

\section{Al}


people with diabetes are significantly at high risk of cancer formation than in the general population $(1,2)$.Both diabetes mellitus and cancer are common chronic diseases which frequently occur in some patients simultaneously.DM is associated with cardiovascular, macrovascular and microvascular complication and there is an increased evidence that DM patients are susceptible to development various cancers(2).

In bladder cancer, the cells lining the urinary bladder lose the ability to regulate their growth and start dividing in an uncontrollable fashion (3). This abnormal growth result in a mass of cells that forms a tumour. Bladder cancer is one of the most common malignancies of the urinary tract which is a continuous anatomical tract, including the kidneys, ureters, and urethra involved in urine formation and excretion $(4,5)$.

The American Cancer Society rate the incidence of bladder cancer and mortality data as estimated to be 73,510 newly diagnosed bladder cancer cases and 14,880 cases that had resulted in death have been projected to occur (5).

The aetiology of bladder cancer is also linked to environmental and chemical exposure to aromatic amines (6), aniline dyes (7), bitumen (8), nitrites and nitrates (9), paints (10) and arsenic (11). The most important environmental factor implicated in bladder cancer is cigarette smoking (12) while the genetic factors are hereditary (6).

A large-scale meta-analysis involving 96 prospective studies showed that DM was associated with a $25-115 \%$ increase in the mortality from cancers involving the / pancreas (13), liver (14), colon and rectum (15). In this study, diabetes mellitus was found to be inversely associated with prostate cancer mortality (16). This and other studies show that
DM has been associated with high risk of cancer $(17,18)$.

The real relationship and biological link between DM and cancers are not fully understood as research works in this area are still on-going globally. Some authorities believe that diabetes mellitus may stimulate carcinogenesis by several mechanisms, such as hyperglycemia, hyperinsulinemia, as well as chronic inflammation (19). More so, DM and cancers both have many common risk factors including hyperlipidemia, obesity, dietary intake, physical inactivity, and exposure to arsenic or bisphenols which are carcinogenic chemicals (20). These obvious risk factors which are common to both may also explain the causal relationship between the two pathologies which are seen to occur simultaneously in certain patients. From the time of the discovery of receptor tyrosine kinase (RTK), and the epidermal growth factor Receptor (EGFR), the understanding of the mechanisms underlying unregulated cellular growth and proliferation has increased dramatically and as a result, RTKs have been widely studied and have become major target for modern cancer chemotherapy in recent times(3). Findings suggest that over-activity of RTK can also lead to cancer. This is as notable as in chronic myeloid leukaemia (21). Type 2 diabetes mellitus is a chronic disease characterized by hyperinsulinemia in its early phase, as beta-cells attempt to overcome tissue insulin resistance. In the later stages of Type 2 DM, many patients are treated with insulin in doses that provide vastly higher serum concentrations than are seen with normal endogenous insulin secretion seen in nondiabetic individuals. Therefore, despite insulin resistance, elevated insulin levels will lead to increased activation of the insulin receptor and may in part explain why people with T2D are at greater risk of developing cancer compared to 
non-diabetic individuals in the general population (22).

The use of an insulin-sensitizers as a first line of drugs in the management and treatment of type 2 diabetes mellitus (T2DM) is widely accepted and may have an advantage over the use of an insulin. Metformin is one such drug and is a safe and inexpensive glucose-lowering drug. 22 Metformin ( $\mathrm{N}^{\prime}, \mathrm{N}^{\prime}$-dimethyl biguanide) is one of the most widely prescribed oral hypoglycemic agents (22). Its mechanism of action is exerted by reducing hepatic glucose production and by increasing insulin sensitivity as well as glucose use by peripheral tissues (22). The use of metformin for the treatment of diabetes was approved in the 1970s in Europe and in 1995 in the United States (22). Metformin use has been gradually increasing with millions of prescriptions filled for patients in the past years. Its use in diabetes has shown to increase overall survival and prevent macrovascular complications better than many other oral anti-diabetic drugs. Many patients use it because of its good compliance rate. Metformin now has a wide variety of indications. It has successfully been used in polycystic ovarian syndrome (PCOS), where insulin resistance is known to cause metabolic disturbances (22). In this setting, it has a favorable effect not only on subfertility but also on cardiometabolic aberrations observed in this syndrome, such as hyperlipidemia and hypertension (22). Metformin can also used in the managing patients with metabolic syndrome and diabetes prevention in high-risk populations (22). Metformin has recently received increased attention for its potential anti-tumorigenic effects that are thought to be independent of its hypoglycemic effects. This has been assessed in multiple studies and is now being tested in clinical trials as an adjuvant to classic chemotherapeutic regimens. Hence the believe that biguanides have anticarcinogenic properties is not new.
Hypothesis

The use of certain diabetic medication put patients at risk of developing bladder cancer. Thus, it is hypothesized that diabetic patients on metformin therapy will have increased risk of developing cancer of the bladder.

Aim

The aim of the study was to assess the effects of metformin therapy on the bladder among diabetic patients in Cape Coast Teaching Hospital.

\section{Epidemiology of bladder cancer}

The global incidence of bladder cancer varies significantly from country to country. With Egypt, Western Europe, and North America having the highest incidence rates, and Asian countries the lowest rates (23). It is the ninth most common cancer worldwide, seventh most common cancer in men and the seventeenth most common cancer in women globally (24, $25)$. It is known that young adults may be affected by bladder cancer, though over $90 \%$ of new cases occur in people of at least 55 years of age (24).

\section{Pathogenesis}

Generally, cancers occur when certain cells in the body start to grow out of the normal control (4). There are different types of cancers in the body. Cancer cell growth. Cancer cells continue to grow and form new, abnormal cells. In most cases the cancer cells form a tumor which may invade other tissues (24). Cells become cancer cells because of damage to DNA. DNA is in every cell and directs all its actions. In a normal cell, when DNA is damaged the cell either repairs the damage or the cell dies. In cancer cells, the repair process is absent .People can inherit damaged DNA, but most often the DNA damage is caused by something in the environment (24). The most identified risk factors for bladder cancer are cigarette smoking and occupational exposure to

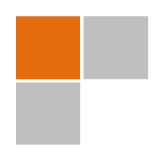


urothelial carcinogens $(26,27)$. Cigarette smoking alone increases the chances of bladder cancer by $50 \%$ in men and $35 \%$ in women(27), while occupational exposure to urothelial carcinogens account for $5-20 \%$ of all bladder cancers (28). The most implicated carcinogens are aromatic amines (e.g. benzidine,2-methyl naphthylamine, 4aminobiphenyl,o-toluidine, and 4-chloro-otoluidine).These are used in rubber, chemical and dye industries $(29,30)$; and also polycyclic aromatic hydrocarbons used in aluminium, coal and roofing industries(31). Bladder cancer has been implicated in painters, hairdressers (32) and exposure to cyclophosphamide (33) and also chronic urinary tract infection (34).

\section{Metformin and Bladder Cancer}

The basis for application of metformin in oncology was first recognized in retrospective epidemiological studies of diabetic patients with cancer. Several observational studies reported decreased cancer incidence and cancer-related mortality in diabetics receiving standard doses of metformin (35). Evans et al. reported a low incidence of risk of subsequent cancer diagnosis in diabetics receiving metformin therapy compared to those patients not receiving the drug), with the protective effect increasing with greater metformin therapy (35). Additional studies examining all forms of cancer have reported reduced cancer risk in diabetics on metformin (compared to those not on metformin treatment) and lower cancer-related mortality in patients receiving metformin compared to those receiving other standard diabetic therapies $(36,37)$.

\section{Methodology}

\section{Study Design and Study Site}

This is a cross sectional study, involving 150 diabetic patients on metformin therapy. Study was carried out at Cape Coast Teaching Hospital, Ghana.

\section{Study Area}

Cape Coast Teaching hospital (formerly called Central Regional Hospital) is a two hundred and twenty six (226) bed referral hospital situated at the northern part of Cape Coast. The hospital, which was the first of a series of ultra-modern regional hospitals established by the Ghana Ministry of Health, started full operations on 12th August 1998. Cape Coast teaching hospital has a diabetic clinic with 1266 diabetic patients yearly. These include 966 old patients and 300 newly diagnosed patients both on metformin and insulin therapy attending the diabetic clinic regularly. The major occupation of people of Cape Coast include: fishing, farming, teaching and trading.

\section{Sample Technique}

Simple random sampling was used to select 150 participants on metformin therapy attending the diabetic clinic at the Cape Coast teaching Hospital.

\section{Study Participants}

One hundred and fifty (150) willing diabetic patients on metformin therapy were used as participants in the study. Information on age, sex, duration of therapy, and occupation were obtained.

\section{Inclusion Criteria}

Diabetic patients who have been on metformin for 4 years and above qualified for participation in this study.

\section{Exclusion Criteria}

Diabetic patients newly diagnosed and those on other forms of therapy aside metformin and people with overt bladder disease were excluded from the study.

Sample collection

Midstream urine samples were obtained after participants were instructed on correct 
mode of urine collection, urine was transported through a suitable medium (cold box).

\section{Sample processing}

Smears were prepared after centrifuging using a test tube containing $10 \mathrm{mls}$ of urine. Supernatant decanted and sediment were resuspended and decanted unto a labelled clean grease-free frosted glass slide. One slide was used to spread the other so that they both have smears on and fixed in absolute ethanol by dipping in a container containing ethanol. It was then air dried. Two slides were prepared for each sample.

After fixation, slides were transferred without drying directly from absolute alcohol then through 95\%alcohol, then through $80 \%$ alcohol, 70\% alcohol, and water to distilled water. It was stained in Harris' hematoxylin for 8 minutes, and gently rinsed in tap water to prevent cells from being washed off. The nuclear staining was differentiated carefully inn $1 \%$ acid alcohol and placed gently in running tap water for 5 minutes.Slides were rinsed in distilled water and transferred through $70 \%$ alcohol, $80 \%$ alcohol, to $95 \%$ alcohol. It was stained with Orange Gold 6 for 2 minutes and rinsed twice in $95 \%$ alcohol before staining with EA50 (Eosin Azure) for 3minutes.After staining with EA50, it was rinsed in 3 changes of $95 \%$ alcohol. Two (2) changes of absolute alcohol 4 changes of xylene and mounted in DPX cover slip was applied and examined microscopically using times 40 objective lens by $a$ cytopathologist.

\section{Examination of slides}

Slide were examined and reported as:

No inflammatory, degenerative, benign or malignant cells were seen. Each slide was read and interpreted twice.

\section{Statistical Analysis}

Data was put into Microsoft Excel and statistical analysis was carried out using
Statistical Package for Social Science (SPSS) for Microsoft Windows, 13th edition.

Ethical Issues/Clearance

Hospital administration protocol was observed and objective of the study was explained to participants. Consent of participants given prior to sample collection. Ethical clearance form IRB/UCC was obtained and clearance given.

\section{Results}

A total of one hundred and fifty (150) participants of ages of 25-86 partook in the study out of which 99 were females and 51 males (see table 1). Peak age group (35.33\%) were between the ages of 51 to 60 , least age group (4.67\%) 80 years and above. Traders of food items and used clothes constituted the peak occupational group of $27.52 \%$, with fishermen $(0.67 \%)$ as least occupation group. Age group of 61 to 70years had the highest duration of therapy of (15.8\%), the least duration of (4.625\%) was among group of 25 to 30 years. The group of 80 years and above had a duration of $(4.857 \%)$.

\begin{tabular}{lcc}
\hline GENDER & COUNT & PERCENTAGE \\
\hline MALE & 51 & $34.0 \%$ \\
FEMALE & 99 & $66.0 \%$ \\
TOTAL & 150 & $100.0 \%$
\end{tabular}

Table 1: Showing the total number of participants and gender in percentage

\section{Discussion}

Ferlay et al estimated high mortality rates among men and women with various types of cancers(23).Evidence shows that patients with diabetes, especially type $2 \mathrm{DM}$ are more susceptible to having a various types of cancers (2,19). However, the incidence of cancer in diabetic patients on metformin 


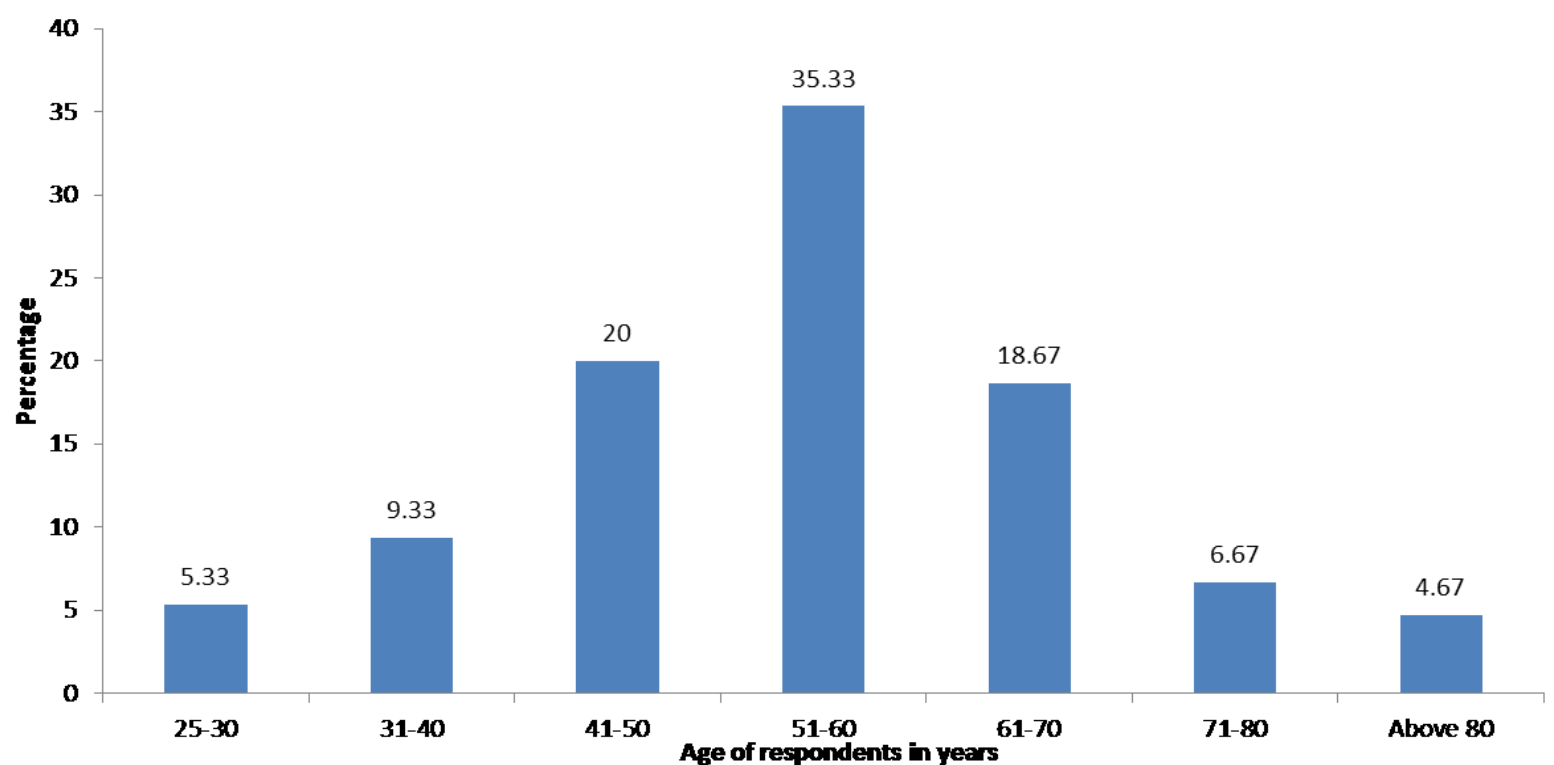

Figure 1: Age group distribution of diabetic patients on metformin therapy

therapy is somehow reduced. Evans et al and Libby et al reported a reduced incidence of cancer rate in diabetics receiving metformin (compared to those patients not receiving the drug), with the protective effect increasing with greater metformin therapy. This implying that those on metformin had reduced cancer rate $(35,36)$. The apparent lower bladder cancer incidence is associated with metformin usage (38).

In this study, the probability of developing bladder cancer among diabetic patients on metformin therapy is $0 \%$ among participants of all categories. No inflammatory, degenerative, benign or malignant cells were observed among urine samples from diabetic patients on metformin. The study found no cytological changes in both men and women. No correlation was observed between the age of diabetic patients on metformin therapy and bladder pathology. About $4.7 \%$ of participants were aged 80 years and above with no abnormal cells (Inflammatory, degenerative, benign or malignant cells) seen microscopically. Although from literature, increase in age puts patients at risk of developing bladder cancer.
The reason for no change in the urothelial lining of the bladder may be attributed to the use of metformin therapy. Such low risk in both sexes may be associated with metformin usage. Many observational studies reported decreased cancer incidence and cancer-related mortality in type 2 diabetics receiving standard doses of metformin therapy $(35,36,37,38,43,44,45)$.

Cancer rate $s$ reduced and is attributed to the following: Metformin has the ability to suppress cancer growth in vivo by activation of LKB1/AMPK pathway, induction of cell cycle arrest and/or apoptosis, inhibition of protein synthesis, reduction in circulating insulin levels, inhibition of the unfolded protein response (UPR), activation of the immune system, and eradication of cancer stem cells (22). Various researchers have propounded that metformin may be associated with a decreased risk of developing cancer and with a better response to chemotherapy $(22,39,40,41)$. These results show that metformin has an anti-proliferative effect associated with cell cycle arrest and apoptosis, which is mediated by oxidative stress, as well as AMPK and FOXO3 activation. This index study further reinforces the potential benefit of metformin in cancer treatment just

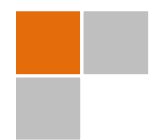


as many other studies (42). Treatment with metformin induces cell cycle arrest in G0-G1 phase and increased cell apoptosis and necrosis, which are associated with increased oxidative stress (42). Pira et al proposed that other factors such as age, gender, work occupation, race etc put people at risk of developing bladder cancer(6).

In this study, both men and women were observed to be at no risk; though literature shows that males are susceptible to bladder cancer. Metformin exhibit direct inhibitory effects on cancer cells (Dowling et al 2011). In this study, some participants had been on metformin for more than 10years, there was no correlation between development of abnormal cells and the duration of therapy hence, it could be said that metformin has no negative effect on the bladder. In a recent study, low doses of metformin $(250 \mathrm{mg} /$ day $)$ findings showed reduced number of rectal aberrant crypt foci (a surrogate marker for colorectal cancer) and decreased the proliferative activity of colonic epithelium in non-diabetic patients(46). Again, analyses of ongoing studies involving neoadjuvant metformin treatment of newly diagnosed breast cancer patients have demonstrated that metformin is safe and well tolerated, and exhibits favourable effects on insulin metabolism and tumour cell proliferation and apoptosis(47).The effects of metformin on cancer cell proliferation have been associated with AMPK activation, reduced mammalian target of rapamycin (mTOR) signalling and protein synthesis, as well as a variety of other responses including decreased epidermal growth factor receptor (EGFR), Src, and mitogen-activated protein kinase (MAPK) activation, decreased expression of cyclins, and increased expression of p27 $(47,48,49,50)$

Recent studies have demonstrated that metformin may also target cancer-initiating cells. For example, metformin inhibited the growth of a subpopulation of breast cancer cells shown to have such property in culture and reduced their ability to form tumours in mice(38).One study on the effect of metformin use on prognosis in urothelial carcinoma of the bladder has been conducted by Rieken et al (51). They concluded that diabetic patients who do not use metformin appear to be at higher risk of cancer-specific and any-cause mortality than patients without diabetic mellitus. It is however uncertain, whether the severity of diabetic mellitus in this group of patients or the use of metformin itself affects outcomes of bladder cancer (51).

The mechanisms by which metformin achieves these effects are still unclear (52). Though is noted that the anticancer effects of metformin are associated with both direct (insulin- independent) and indirect (insulindependent) actions of the drug. The indirect, insulin-dependent effects of metformin are mediated by the ability of AMPK to inhibit the transcription of key gluconeogenesis genes in the liver and stimulate glucose uptake in muscle, thus reducing fasting blood glucose and insulin (53). Metformin also reduces circulating insulin levels by $22 \%$ and improves insulin sensitivity by $25 \%$ in non-diabetic women with breast cancer, highlighting the insulin-lowering effects of metformin as a potential mechanism of action in the treatment of breast cancer (47).

\section{Conclusion}

Metformin is a widely prescribed antidiabetic drug with an established efficacy coupled with a high safety profile and affordability and it has also been associated with good outcomes in cancer patients (54).

Conclusive evidence has shown that metformin therapy has no additive effect on the development of bladder cancer among diabetic patients. All categories of study participant showed no cytological change in the urothelial lining of their bladder. Moreover, in a similar study of diabetic prostate cancer 
patients, metformin use was not associated with benefit (55). Thus, further clinical research is needed to fully evaluate the impact of metformin on cancer of the bladder.

\section{Limitations}

The absence of a control group from diabetic patient on other form of diabetic therapy aside metformin and those on no therapy at all. Similar study may be conducted using a bigger sample size in other regions and other countries.

\section{Recommendation}

We recommend that metformin therapy should still be used in the management of diabetes since it has no negative effect on the urothelial lining of the bladder.

\section{Conflict of interest}

The authors declared no conflict of interest.

\section{References}

1. Edward G, Daniel H, Michael A, Richard MB, Susan G, Laurela H, Michael P, Judith G, Douglas M. Diabetes and cancer. Diabetes Care. 2009;33: $1674-85$.

2. Chowdhury TA. Diabetes and Cancer. QJM. Journal of the Association of Physician 2011;103: 905-915.

3. www.nih.gov(Bladder cancer)

4. Marriane BY, Susan AO, Ulka BC, Carol BK. Incidence of cancer with type 2 diabetes. Diabetes and metabolic syndrome.2009; 3612-16:

5. Siegel R, Nashadham D, Jemal A. cancer statistics. Cancer J Clin 2012;62:10-29.

6. Pira E, Pislatto G, Negri E, Romano C, Boffetta et al. Bladder cancer mortality and workers exposed to aromatic amines: A 58 year old follow up. J National Cancer Inst. 2010;102: 1096-1099.

7. Bulbuyan MA, Figgs LW, Zahn SH, Savitskaya T, Goldford A. Cancer incidence and mortality among betanaphtalamine and benzidine dye workers in Moscow. Int. J. Epidemol 1995;24: 266-275.

8. Behrens L, Schill W, Ahrens W. Elevated cancer mortality in a German cohort of bitumen workers. J. Occup Environ Hyg. 2004;6: 555-561.

9. Fermici LM, Sinha R, Ward MH, Graubard BL, Hollenbeck AR. Meat and components of meat and the risk of bladder cancer in the NIH-AARP. Diet and health study. CANCER 116:4345-4353.

10. Guha N, Steenland NK, Merleth F, Altier A, Cagliano V. et al. Bladder cancer in painters: A meta analysis. Occup Environ Med. 67: 568-573.

11. Fernandez MJ, Lopez JF, Vilvadi B, Coz F, long term prosper of arsenic in drinking water in bladder cancer health care and mortality water 20 years after end of exposure. J UROL 2012; 187: 856-861.

12. Freedman ND, Siverman DT, Hollenbeck AR, Schatzkin A, Abret CC. Association between smoking and risk of bladder cancer among men and women. JAMA 2011; 306: 737-745.

13. Ben Q, Xu M, Ning X, Liu J, Hong S. et al. Diabetes mellitus and the risk of pancreatic cancer. Eur J Cancer 2011; 47: 1928-1937.

14. Wang C, Wang X, Gong G, Qui W. et al. Increase risk of hepatocellular carcinoma in patients with diabetes mellitus. Int J Cancer. 130: 1639-1648.

15. Yuhara H, Steinman C, Cohen SE, Corley DA, Tei Y. et al. Is diabetes an independent risk factor for colon cancer and rectal cancer. Am J Gastroenterol 2011;106: 1911-1921. 
16. Kasper JS, Giovannuci E. A meta analysis of diabetes mellitus and risk of prostate cancer. Cancer Epidermiol Biomarker Prev. 2006;15:2056-2062.

17. Cheng KC, Chen YL, Pang YT, Sung FC. Risk of oesophagus cancer in diabetes mellitus-A population based study in Taiwan. BMC Gastro enterol 2012,12: 777.

18. Ekpe EL, Omotoso AJ. Diabetes mellitus and the risk of cancer formation: Possible link and review of literature. American journal of Internal Medicine 2015;3(4):180-184

19. Giovannucci E, Hallan DM, Archer MC, Bergenstal RM, Gapstor SM, Habel LA, et al. Diabetes and cancer: A consensus report. Diabetes Cares 2010;33(7): 1674-85:

20. Tseng CH, Tseng FH. Diabetes and gastric cancer -the potential link. World J Gastroenterol 2014; 20(7):1701-1711.

21. Ahmad Shazaib, Chowdhury TA, Boucher BJ.Diabetes and cancer:Could Vitamin D provide the link? Journal of Diabetes and its complications.2012;10(5).

22. Kourelis TV, Siegal RD, metformin and cancer: new application for an old drug. Med. Oncol. 29(2). 1314-27.

23. Ferlay J, Randi G, Bossett C, Loui F, Negri E et al. Declining mortality from bladder cancer in Europe. BJU Int 2008;10(1): 11-9:

24. Colombel M, Soloway M, Akasa H, Bohle A. et al epidemiology, staging grading and risk stratification of bladder cancer. European Urology. 7(10): 618-626.

25. Murta-Nascimento , BJ Schmitz-Drager ,MP Zeeger et al. Epidemiology of urinary bladder cancer from tumor development to patient health. World J Urol. 2007;25:285-295.

26. PJ McCarthy, CA Harris, E Neal. The accuracy of recording of occupational history in patients with bladder cancer. Br J Urol 1997;19: 91-93. 1997.

27. MP Zeegers, FE Tan, Dovant PA, van den Brendt. The impact of characteristic of cigarette smoking on urinary tract cancer risk. CANCER 2000;89: 630-639.

28. $P$ Veneis, $L$ Simonato, Proportion of lung and bladder cancer of male resulting from occupation: A systematic approach. Arch Environ Health. 1991;46: 6-15.

29. Chung KT. The etiology of bladder cancer and its prevention.J Cancer Sci Ther 2013;5(10):346-361.

30. Chung KT. The etiology of bladder cancer and its prevention.J Cancer Sci Ther 2013:5(10):346-361.

31. Boffetta P,Jourenkora N,ustavasson. Cancer risk from occupational and experimental exposure to polycyclic aromatic hydrocarbons. Cancer causes and control 8:444-472.1992

32. Golka K, Wiese A, Assenato G, Bolt M. Occupational exposure and urologic cancer. World J Urol 2004;21:382-391.

33. Travis LB,Curtis RE, Glimelius et al.Bladder and kidney cancer following cyclophosphamide therapy from nonHodgkins lymphoma.J Natl Cancer Inst 1995;87:524-30.

34. Kanto AF, Hartge P, Hoover RN, et al. Urinary tract infection and risk of bladder cancer. Am J Epidermol 1984;119:510-515.

35. Evans JM, Donnelly LA, Emshie-Smith AM, Alessi DR, Andrew Morris. Metformin and reduced risk of cancer in diabetic patients.BMJ 2005;330(503):1304.

36. Libby G, Louis Donnelly,Peter Donan, Dario Andrew Morris, Josie MM. New uses of metformin are at low risk of incident cancers. Diabetes Care 2009;32:1620-1625.

37. Hirsch HA,Dimitrious I,Tsichlis PN,Struhl K. Metformin selectively targets cancer stem cells and acts together with chemotherapy to block tumor growth and prolong remission. Cancer Res 2009;69(190):7507-7511.

38. Bowker SL, Marjumdar SR, Veugester P, Johnson J. Increased cancer - related mortality for patients with type 2 diabetes who use sulphonureas or insulin - Diabetes Care 2006;29(2): 254-258.

39. Shackelford DB, Shaw RJ. The LKB1-AMPK pathway: metabolism and growth control in tumor suppression. Nat Revl Cancer. 2009;9(8): 567-573. 
40. Zakikhani M, Dowling R, Fantus TG, Sonenberg N, Pollak M. Metform is an AMP kinase - dependent growth inhibitor for breast cancer cells Cancer Res. 2006;16(2): 10269-73.

41. Zakikhani M, Dowling R, Fantus TG, Sonenberg N, Pollak M. The effect of adiponectin and metformin on prostate and colon neoplasia involve activation on AMP-activated kinase. Cancer Prev. Res.2008; 1(5): 369-75.

42. Quieroz EA, Puukila S, Eichler R, Sampaio SC et al. metformin induces apoptosis and cell cycle arrest mediated by oxidative stress, AMP and FOXO 3a in MCF. 7 breast cancer cell. Plos One. 2014;9(5): E 982.

43. Dowling Ryan JO, Goodwin PJ, Stambolic V. Understanding the benefit of metformin use in cancer treatment. BMC Med. 2011;9(33):

44. Scarpello JHB, Howlett HCS. Metforum therapy and its clinical uses. Diabetes and vascular disease research. 2008;5(3)

45. Bradford SA, Khan A. individualizing chemotherapy using the anti-diabetic drug metformin as an adjuvant: an exploratory study. Cancer Sci. Ther 2013;5(3)

46. Hosono K, Endo H, Takahashi H, Sugiyama M, Sakai E, Uchiyama T, Suzuki K.Metformin suppresses altered aberrant cancer crpts foci in a short term clinical trial.Cancer Rev Res 2010;36:1077-1083.

47. Dowling JO, Goodwin PJ, Stambolic. Understanding the benefit of metformin use in cancer treatment .BMC Medicine 2011;9:33

48. Zhang Tao,Zhan Yinan, Guo Peng, Xiong Hui et al.The anti-diabetic drug metformin inhibits the proliferation of bladder cancer cells in vitro and in vivo. Int J Mol Sci 2013;14(12):24603-24618.

49. Bennett AM, Tonks N. Regulation of distinct stages of skeletal muscle differentiation by mitogen-activated protein kinase.Science 1997;278(5341):1288-1291.

50. Lui Tsan Zon, Chen Chung Y, Yiin Shuen et al. Molecular mechanism of cell cycle blockage of hepatoma cells through suppression of mitogen-activated protein kinase. Food and Chemical Toxicology 2006;446:227-235.

51. Rieken M, Xylinase E, Kluth L, Crivelli JJ et al. Effect of diabetes mellitus and metformin use of oncologic outcome of patients treated with radical cystectomy.Urol Oncol 2013;32(1):7-49.

52. Pulito C, Rana TP, Sanli T, Muti P, Blandino G. Metformin: On going journey across diabetes, cancer therapy and prevention.Metabolites 2013;3(4):1051-1075.

53. Witters LA.The blooming of French lilac. J Clin Invest.2001;108:1105-1107.

54. Rizos C , Elisaf MS. Metformin and cancer. Eur J Pharmacol 2013;705(1-3):96-105.

55. Patel T, Hruby G, Badani K, Abale Shen C, Mckierman JM. Clinical outcomes after radical prostatectomy in diabetics patients treated with metformin. Urology 2010;76:1240-1244. 